\title{
Innovative Use of Media to Increase Student Engagement for a Large Second-year Core Course: "Engineering Economics"
}

\author{
Scott A.C. Flemming, M.A.Sc., P.Eng. \\ Instructor, Dalhousie University \\ Scott.Flemming@dal.ca
}

\begin{abstract}
As most practitioners are aware, student engagement in large first- or second-year engineering classes is difficult. In a traditional lecture-style presentation instructors are given the challenging task of explaining difficult technical material to several hundred students in such a way that they are not distracted by their friends, cellphones, or the lecture hall atmosphere. In the literature, various solutions to student engagement are suggested: flipped classrooms, design projects, brainstorming sessions, paraphrasing exercises, and selfrating exercises [1]. The author attempted to implement various of these interventions with little anecdotal success. However, a modification of the "think-pairshare" idea as described by Karl Smith, from a subjective point of view, seemed to capture the class more than the default lecture/powerpoint method. Enumeration of student comments about the intervention and a comparison of means from student self reports of "stimulation of learning" suggests that the intervention was successful. Future work is planned to further refine the lectures in terms of student engagement in the lecture theatre and the tutorial classroom.
\end{abstract}

Keywords: Student engagement; large class-size; lecture style; content retention; stimulation of learning.

\section{INTRODUCTION}

Many of we practitioners understand the difficulty in engaging large classes first-hand but Felder et al. [3] have done the work of putting together a literature review of common issues in large classes and interventions that are meant to mitigate this problem.

\subsection{The Problem}

Educational methods differ depending on the context. Felder et al. suggest that, as far as engineering is concerned key goals are to sharpen problem-solving, critical thinking skills, and encourage long-term retention of relevant facts [3]. This is opposed to the goals of disseminating large volumes of data for short-term retention. For the latter traditional one-way lecture-style communication is successful. For the former active- and student-centred learning is desired [3]. Felder quotes a result from a key study by McKeachie that found that immediately following a 50-minute lecture, students could recall $70 \%$ of that which was taught in the first ten minutes but only $20 \%$ of that which was covered in the last 10 minutes [3]. A study by Tiwari et al [4] showed that medical students engaged in problem-based learning performed significantly better than their peers who were engaged in lecture-based learning according to various critical-thinking skills up to even two years after their learning experience. The conclusion is simple but important: we must begin to adopt different teaching styles to prepare engineering students to be successful in the future.

\subsection{Suggested Solutions}

Active learning seems to be a more effective tool in the context of engineering education. How then should we change our teaching practice? The Large Classroom Engagement Handbook published online by McMaster University suggests various interventions to make the lecture-hall a more engaging environment [1]. For example:

- Brainstorming

- Directed paraphrasing

- Electronic polling

- The one-minute paper

- Self-rating of concept knowledge

- Think-Pair-Share

The author attempted a few of these methods and, subjectively speaking, they did not seem to change the typical behaviour of students. If students were asked to turn to their partner or sit by themselves and perform some task (brainstorm, self-rate, or paraphrase), it appeared as if most were not doing so and simply using that time to check their email or social media. Electronic polling was also used but some technical issues (slow response times, inability to login to the app, etc) seemed to slow the flow of the class and cause anxiety for the students around getting participation marks.

All this being said, the "think-pair-share" idea seemed to be the most likely to bear fruit. Although the author tried a few different variations of this without much luck, a paper by Smith [2] began an idea that seemed to resonate with the students. 


\section{INTERVENTION USED}

According to the "Large Classroom Engagement Handbook" the Think-Pair-Share (TPS) procedure is as follows [1]:

1. Give students a problem

2. Ask each student to develop a step-by-step plan to solve the problem

3. Ask students to form groups of two or three and compare one another's plans

4. Ask them to discuss and refine a "best" plan

5. Have the students "solve" the problem

6. Ask for feedback from the groups to share with the class as a whole

Now this approach is difficult to utilize in the Engineering Economics course insofar as there aren't necessarily a significant amount of "problems" to be solved where students need to brainstorm to think of creative approaches. Solution approaches in this class are rather regimented and the calculations are such that there are rather few methods that will lead to a "correct" answer. Such an approach as above may be more useful in a first- or second-year design course where creative solutions to particular problems make more contextual sense.

Although the approach itself was difficult to implement for Engineering Economics, the idea as described by Smith [2] encouraged new thought on the subject. His take on the TPS method was to break a traditional 50-minute lecture into several pieces interspersed with students engaging one another. First, he suggested a brief introduction/agenda for the class followed by a 10-12 minute lecture. At this point students would form groups and discuss the content something like described in the TPS method above for 3-4 minutes. Then he would give another 10-12 minute lecture followed but another 3-4 minute TPS activity. Finally he would end with a 5-6 minute summary of the content covered that day and any feedback/information given to him by the students. Perhaps what Smith was trying to do here was exploit what many practitioners know in their "gut" and was reported in the study by McKeachie above: that student attention and information retention is optimal only for about 10 minutes. It seems that his idea of incorporating useful "breaks" into his lecture meant to exploit that.

As mentioned above, although this particular intervention was not used as prescribed, it made the author think about other similar interventions that could be used to achieve the same effect. The idea that came was that of using a video "case study" to make a tangible point of what the more somewhat abstract class material was trying to explain.

\subsection{Course Context}

For those not familiar with Engineering Economics what follows is a brief explanation of the course. The course could be called "Engineering Finance" but it is not due to various histories, conventions, and academic programs now tied to the current nomenclature. That being said it is important to realize that Engineering Economics is not really about economics in the academic sense. It is not about supply and demand curves, market equilibrium, or inelastic or elastic commodities. In that way the name is very misleading. The course is essentially about finance. And therein lies that challenge of teaching the course - there are not a list of difficult concepts for students to grasp; there is really only one key concept to understand: the "time-value of money." That is, $\$ 100$ today is worth more than $\$ 100$ a year from today since one could invest that initial $\$ 100$ and make a return on it, say $10 \%$ (and so be left with $\$ 100$ a year later). After students understand this concept, the rest of the course is really the mechanics of applying that central idea to various different scenarios.

Like any course, this course can be academically challenging but the challenges are different than the standard course. It is mainly that that students have to think very differently than they do in engineering contexts and that the key concept in this course, although rather simple, has sometimes unexpected ramifications. A key difference is that most of their courses are dealing with describing and understanding natural phenomena and this course deals with a human-made phenomena: the "time value" of money.

\subsection{Variation of Smith's idea}

To come back to the key point of this paper, the intervention chosen for this particular course was not to ask students to TPS, but rather to introduce a short video clip in the centre of each class. It was not a replica of Smith's method [2] but a modification of it. The author chose to have a short introduction to each class, lecture for 20-22 minutes, show a 5-minute video clip, and then lecture for another 20-22 minutes. In some ways a very different intervention but the similarity was to introduce a "break" into the class - a change in information medium that did not give the students as easy a chance to "check out" by using their phones to answer emails or social media messages.

It may be somewhat counter-intuitive, but after trying over several classes in past years for students either to discuss with a partner some new topic and for this year to get students to watch a movie - strangely students appeared more engaged with a screen than with their peers (perhaps a sad comment on our current social reality). The positive was that when the movie was running, there were more eyes looking at the big screen 
and engaged in the particular content the author had prepared for them rather than their own personal phone or tablet screens.

The author should share at this point that the lecture theatre was actually a movie theatre. While campus construction is taking place, large first- and second-year courses have been moved to the $300+$ seat movie theaters nearby. Although at first it seemed like a liability, there was a real opportunity to take advantage of the fact that the Instructor had access to a very high-quality projector and a wealth of fair-use videos available on YouTube or Vimeo (provided such video's have been posted by the copyright holder.) "If you can't beat 'em, join 'em" is the mantra that comes to mind.

\subsection{Examples of Content}

Now the question arises - what sort of content was chosen and why? Since the course was not highly theoretical but purposed to introduce engineering students to best practices in money management, it was believed that case studies would be the most relevant content to show. A common question/comment (or rather frustration) of students with regards to this finance course is "Why do we have to take this anyway? It has nothing to do with engineering." It was these questions the author wanted to address along with questions of Ethics and Sustainability. The videos were meant to address these questions and state, "Here is an example as to why understanding fiscal decision-making principles is important with regards to successful business operation, successful environmental stewardship, and successful social responsibility."

The case studies encompassed a wide variety of topics, from ethics, sustainability, to stories of engineers who wanted to break out of their current jobs and start their own businesses. During this course the author, for the first time in teaching the content, was receiving emails from students sharing their interest in some of the case studies and questions related to them as well as students offering links to similar content they had seen elsewhere and thought the instructor could use for future years.

To introduce the idea of "engineering finance" the first video shown is one about the history of Easter Island. In this video the instructor tries to put the course in a big picture: the method we use to decide how we spend our resources is very important. The way this video summarizes the history of Easter Island is that the method in which the inhabitants managed (or mismanaged) their (financial) resources resulted in the desolation of the island and its inability to sustain the population living on it. The point of this lecture was to state that finance is not a-moral and that we must understand that financial analysis can help us make decisions but cannot tell us if what we are doing is right or wrong (although the outcome of such analysis is often a "go" or "no-go" result which suggests that the method does indeed give us a "right" answer.)

One of the student's favourite videos is the "John Oliver" lecture [6]. As we begin to cover the section on effective interest rates the instructor plays a video from "Last Week Tonight with John Oliver." Here Mr. Oliver does a magnificent job of highlighting a frustrating social issue in a very entertaining (and of course engaging) way. This keeps the students engaged for a sufficient amount of time that we can discuss the assumptions and calculations behind some of the claims that patrons are being charged $500+\%$ on their "payday" loans and discuss the legality of such financial "products."

A last example in the environmental front is that of a very low-footprint company in the UK called GroCycle that takes nutrition-rich waste coffee grinds and turns them into the fodder for gourmet mushrooms grown in urban warehouses [7]. The point of this video was to show students that "green" companies are indeed financially viable and that it is possible to do business differently in our current market-driven environment and still survive and even thrive.

\section{OUTCOMES}

There are two more concrete ways (as opposed to intuition) in which the author attempted to validate the approach of changing the medium of information. One of those ways is through comments of students left in their usual end-of-term Student Ratings of Instruction. It is interesting to note that of all of the 13 comments left by students mentioning the word "video" or "movie" were all positive in nature. Some examples follow are answer to the question "What did the Instructor do to help your learning in this class?"

-He kept me engaged. I very much so appreciated the 2-5 minute videos that incorporated what we were learning to the real world that we would watch about half way through the class. It kept everyone engaged and awake after sitting there for so long."

- Movie of the day was interesting and provided information on how the work we are learning is applicable to the real world."

- "Related course topics to things that we are interested in today with videos/guest speakers."

- "In each class, [the instructor] would show the class a video relating to the topic being discussed during that specific lecture, and also to real financial matters in the world."

- "The videos provided a great recess from our lecture marathon."

- "I appreciated the fact that he introduced unique content in the form of YouTube videos or a case study." 
"He uses a youtube clip in each lecture when he feels the class slipping and it keeps us engaged."

> "Movie time is the best time!"

- "The video breaks during lecture keeps me awake to continue concentrating in class."

Although only 13 of the 93 respondents mentioned the use of the videos in their SRIs, all of those 13 did so in a positive light.

The last method of validation is in the form of the numerical SRI responses with regards to scores for "Stimulation of Learning." When performing a hypothesis test of whether or not the mean rating for this course was equal to the average rating for the department, the null hypothesis was rejected. Over the three years of teaching this course the rating of "Stimulation of Learning" was less than that of the department average until the final year where the videos were brought in.

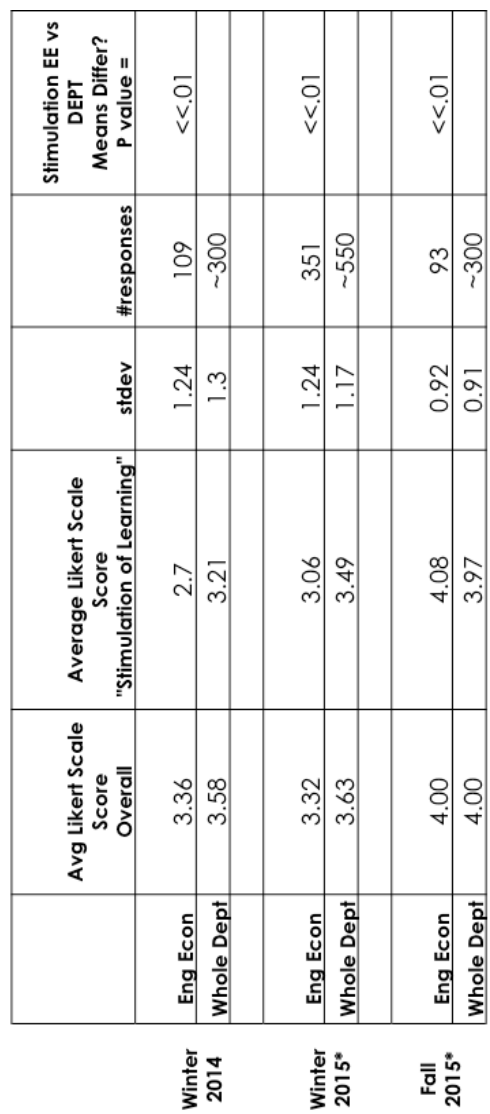

Figure 1 - SRI Hypothesis Test

\section{LIMITATIONS}

This paper is an account of a particular technique in action not a systematic review of the literature in active learning or use of media in a teaching and learning setting. It is also not a research study in a highly controlled environment. As such its generalizability to other contexts is limited.

\section{CONCLUSIONS AND FUTURE WORK}

The conclusion of this paper is that student engagement was increased by use of videos in the midst of a traditional lecture-style class. This conclusion is supported by Student Rating of Instruction data - both in written and numerical form. In the future, the instructor would like to do a thorough literature review of the use of video in the classroom as well as explore how to encourage engagement in the tutorial sections of this class.

\section{Acknowledgement}

The author would like to thank Dalhousie University's Centre of Learning and Teaching which generously funded the ability to present at CEEA 2017 through the "Change one Thing Award" grant.

\section{References}

[1] "Large Classroom Engagement Handbook" http://www .eng.mcmaster.ca/fda/teaching.html. Accessed on February 9, 2016.

[2] Karl A. Smith, Going Deeper: Formal Small-Group Learning in Large Classes. New Directions for Teaching and Learning, no. 81, Spring 2000.

[3] Richard M. Felder et al., The Future of Engineering Education II. Teaching Methods that Work. Chem. Engr. Education, 34(1), 26-39 (2000).

[4] Agnes Tiwari, Patrick Lai, Mike So, and Kwan Yuen. A comparison of the effects of problem-based learning and lecturing on the development of students' critical thinking. Medical Education, 40, 547-554, (2006).

[5] "Lessons from the past." YouTube Video, 11:45. Posted by GreenovateChina. Upload 17 Feb 2012.

https://www.youtube.com/watch? $\mathrm{v}=\mathrm{jYliCfzOkDE \& t}=573 \mathrm{~s}$

[6] "Predatory Lending: Last Week Tonight with John Oliver." YouTube Video, 16:31. Posted by LastWeekTonight. Upload 10 August 2014. https://www.youtube.com/watch?v=PDylgzybWAw

[7] "GroCycle Urban Mushroom Farm - Growing Gourmet mushrooms from waste coffee." YouTube Video, 2:26. Posted by FungiFutures. Uploaded 15 Aug 2014. https://www.youtube.com/watch?v=ID_Y4IgOWdI 